\title{
What is primary care anyway?
}

Primary care is a favourite within certain circles in today's practice of medicine. In particular Health Maintenance Organizations (HMOs) look at it as one of the major strengths against the growth of technology driven increase in medical care. It is also seen at certain political levels as one way to provide medical care to everyone in the community irrespective of income or social level.

My personal view is that the promotion of primary care by politicians should be met with ambivalence, since they would like to explore this avenue as a solution to health care problems.

I believe also that primary care as a medical discipline must exert control over its own future and mistakes.

What is primary care anyway? My understanding is that effective primary care refers to the extent to which the needs of the population are met. One can see variations from certain norms in clinical procedures but never should intervention be encouraged with the aim of satisfying patients demands rather than the real needs. The main thrust is that primary care must be directed at the needs rather than the demands of the patients. It is also my understanding that the current level of training of primary doctors in health care can not give to them the ability to cope with all health problems in the community irrespective of the economics of the patients. Although the dissemination of information can help to improve the decision making for general practitioners, specialized medicine is the only pathway to assure proper care in complicated cases and long term care in chronic diseases. My current thinking on this matter, sometimes acting as a primary care doctor and at other times in secondary care, is that only interactive medicine will guarantee effective care. The political and financial view of primary care doctors as "gate keepers" of the level of practice will only allow cost reduction at the expense of proper care.

Primary care doctors treat a variety of illness in any one patient, in contrast to secondary care where many patients are treated for the same illness. However, the proper level of training is not there to treat everything and therefore one might choose the wrong treatment. Promotion of primary care should be done in parallel with secondary care and we may have doctors who can practise both.

\footnotetext{
* M.D., P.h.D., F.A.C.P. 